\title{
Sex differences in lipid and glucose kinetics after ingestion of an acute oral fructose load
}

\author{
Christel Tran ${ }^{1}$, Delphine Jacot-Descombes ${ }^{1}$, Virgile Lecoultre ${ }^{1}$, Barbara A. Fielding ${ }^{2}$, Guillaume Carrel $^{1}$, \\ Kim-Anne Lê ${ }^{1}$, Philippe Schneiter ${ }^{1}$, Muriel Bortolotti ${ }^{1}$, Keith N. Frayn ${ }^{2}$ and Luc Tappy ${ }^{1,3 *}$ \\ ${ }^{1}$ Faculty of Biology and Medicine, Department of Physiology, University of Lausanne, Rue du Bugnon 7, Lausanne CH-1005, \\ Switzerland \\ ${ }^{2}$ Oxford Centre for Diabetes, Endocrinology and Metabolism, University of Oxford, Oxford, UK \\ ${ }^{3}$ Service of Endocrinology, Diabetes and Metabolism, CHUV, Lausanne, Switzerland
}

(Received 17 November 2009 - Revised 25 March 2010 - Accepted 13 April 2010 - First published online 14 June 2010)

\begin{abstract}
The increase in VLDL TAG concentration after ingestion of a high-fructose diet is more pronounced in men than in pre-menopausal women. We hypothesised that this may be due to a lower fructose-induced stimulation of de novo lipogenesis (DNL) in pre-menopausal women. To evaluate this hypothesis, nine healthy male and nine healthy female subjects were studied after ingestion of oral loads of fructose enriched with ${ }^{13} \mathrm{C}_{6}$ fructose. Incorporation of ${ }^{13} \mathrm{C}$ into breath $\mathrm{CO}_{2}$, plasma glucose and plasma VLDL palmitate was monitored to evaluate total fructose oxidation, gluconeogenesis and hepatic DNL, respectively. Substrate oxidation was assessed by indirect calorimetry. After ${ }^{13} \mathrm{C}$ fructose ingestion, 44.0 (SD 3.2) \% of labelled carbons were recovered in plasma glucose in males $v .41 .9$ (SD 2.3) \% in females (NS), and 42.9 (SD 3.7 ) \% of labelled carbons were recovered in breath $\mathrm{CO}_{2}$ in males v. 43.0 (SD 4.5) \% in females (NS), indicating similar gluconeogenesis from fructose and total fructose oxidation in males and females. The area under the curve for ${ }^{13} \mathrm{C}$ VLDL palmitate tracer-to-tracee ratio was four times lower in females $(P<0.05)$, indicating a lower DNL. Furthermore, lipid oxidation was significantly suppressed in males (by 16.4 (SD 5.2$), P<0.05)$, but it was not suppressed in females $(-1.3(\mathrm{SD} 4.7) \%)$. These results support the hypothesis that females may be protected against fructose-induced hypertriglyceridaemia because of a lower stimulation of DNL and a lower suppression of lipid oxidation.
\end{abstract}

De novo lipogenesis: VLDL TAG: Endogenous glucose production

The metabolic effects of a high-fructose diet have been widely studied in both animals and human subjects ${ }^{(1)}$. High-fructose diet is associated with the development of at least two features of the metabolic syndrome, i.e. insulin resistance and increased plasma VLDL TAG. The latter may in turn be associated with an increased production of small, dense LDL particle with high atherogenic potential ${ }^{(2,3)}$.

It has been reported by several authors that a high-fructose diet increases plasma TAG less and does not reduce insulin sensitivity in both pre-menopausal women and female rodents compared with males $^{(4-11)}$. This may be due to a sex-specific difference in either fructose metabolism or adaptations to chronic fructose overfeeding. To our knowledge, whether the metabolism of an acute fructose load differs in females and males has not been specifically assessed. Fructose disposal relies mainly on the stimulation of carbohydrate oxidation (fructose oxidation in splanchnic organs and indirect oxidation of glucose and lactate synthesised from the fructose), gluconeogenesis, hepatic glycogen storage and hepatic de novo lipogenesis (DNL). The latter, although a quantitatively minor pathway for fructose disposal, may be closely linked to hepatic VLDL TAG production and synthesis of intra-hepatic lipids. Therefore, we hypothesised that fructose-induced stimulation of DNL may be blunted in pre-menopausal women.

To evaluate this hypothesis, we compared the metabolic fate of ingested fructose in healthy male and female human subjects. To gain insight into the pathways used for fructose metabolism, ingested fructose was labelled with ${ }^{13} \mathrm{C}_{6}$ fructose, and incorporation of ${ }^{13} \mathrm{C}$ into breath $\mathrm{CO}_{2}$, plasma glucose and plasma VLDL palmitate was monitored to evaluate total fructose oxidation, gluconeogenesis and hepatic DNL, respectively.

\section{Subjects and methods}

\section{Subjects}

Nine healthy young male $(25 \cdot 1$ (SD 5.1) years) and nine healthy pre-menopausal female (23.8 (SD 4.8) years) subjects participated in the present study (Table 1). All the subjects were in apparent good health, had a BMI between 19 and

Abbreviations: AP, atom percent; DNL, de novo lipogenesis; EGP, endogenous glucose production; FFM, fat-free mass; FM, fat mass; Ra, rate of glucose appearance; TTR, tracer-to-tracee ratio.

* Corresponding author: Professor Luc Tappy, fax +41 69255 95, email luc.tappy@unil.ch 
Table 1. Anthropometric data of subjects at baseline

(Mean values and standard deviations, $n 18)^{*}$

\begin{tabular}{lccccc}
\hline & \multicolumn{2}{c}{ Males $(n 9)$} & & \multicolumn{2}{c}{ Females $(n 9)$} \\
\cline { 2 - 3 } \cline { 6 - 7 } & Baseline, mean & SD & & Baseline, mean & SD \\
\hline Age (years) & 25.1 & $5 \cdot 1$ & & 23.8 & 4.8 \\
Weight $(\mathrm{kg})$ & 73.7 & 9.0 & & $62.4 \dagger$ & $6 \cdot 1$ \\
BMI $\left(\mathrm{kg} / \mathrm{m}^{2}\right)$ & 22.1 & 1.8 & & 22.2 & 1.3 \\
Fat-free mass (\%) & 75.4 & 4.5 & & $68.9 \dagger$ & 5.0 \\
Fat mass (\%) & 24.5 & 4.5 & & $31.1 \dagger$ & $5 \cdot 1$ \\
\hline
\end{tabular}

* Anthropometric data were compared in both sexes using a two-sided Wilcoxon rank sum test for unpaired values.

t Mean values were significantly different from the corresponding values in male subjects $(P<0.05)$.

$25 \mathrm{~kg} / \mathrm{m}^{2}$, and were moderately physically active $(<3 \mathrm{~h} /$ week $)$. They were non-smokers, and were not taking any medications at the time of the study. None of the women were on birth control pills. Female subjects were tested during the follicular phase (days 1-10) of their menstrual cycle. The present study was conducted according to the guidelines laid down in the Declaration of Helsinki, and all procedures involving human subjects/patients were approved by the ethical board of Lausanne University School of Biology and Medicine. Written informed consent was obtained from all the subjects.

\section{Anthropometric and body composition measurements}

At inclusion, standing height was measured using a stadiometer. Since bioelectric impedance was not available at the inclusion site, fat mass (FM) and fat-free mass (FFM) were estimated from the following skinfolds: biceps, triceps, suprailiac and subscapular ${ }^{(12)}$. All the measurements were obtained on the right side, with the subject upright and relaxed. The complete set of measurements was carried out by a single operator and performed three times. Preparation of diets, fructose supplementation and the ${ }^{13} \mathrm{C}$ fructose loads administered during the test were based on these measurements.

Body composition was measured on the day of the metabolic test by bioelectrical impedance using the BC-418 eight-contact electrode system (Tanita Corporation, Tokyo, Japan). FFM and FM were calculated using the manufacturer's equations. All the calculations pertaining to the metabolic tests were done using the value of FFM obtained by bioelectrical impedance.

\section{Study design and diet}

Each subject was studied on one occasion. During the $3 \mathrm{~d}$ preceding the metabolic test, subjects received a standardised, weight maintenance diet (total energy intake $=1.6$ times the resting energy requirements calculated with the equation of Owen; $15 \%$ proteins, $30 \%$ lipids and $55 \%$ carbohydrate (simple sugars 10\%)). During this period, all the foods were provided as pre-packed, weighed portions for each meal. Three meals and two snacks were provided each day, while subjects were asked not to consume any other food or drinks (including alcoholic beverages and coffee).
After this period of controlled diet, subjects were studied for $2 \mathrm{~h}$ in the post-absorptive state (time $-120-0 \mathrm{~min}$ ) and over $6 \mathrm{~h}$ period after fructose ingestion (time $0-360 \mathrm{~min}$ ).

\section{Metabolic investigations}

Subjects reported at 07.00 hours to the metabolic unit of the Lausanne University Hospital after a $10 \mathrm{~h}$ fast. On arrival, the subjects were asked to void, and body composition was estimated by bioelectrical impedance (Tanita Corporation). Thereafter, they rested on a bed in a semi-recumbent position until the end of the test. A catheter was inserted into an antecubital vein for collection of blood samples. The hand on the same side was kept in a thermostabilised box heated at $50^{\circ} \mathrm{C}$ to achieve partial arterialisation of venous blood. Another catheter was inserted into an antecubital vein of the contralateral forearm for $6,6-{ }^{2} \mathrm{H}_{2}$-glucose and ${ }^{2} \mathrm{H}_{5}$-glycerol infusions. Whole-body glucose and glycerol turnover was assessed using infusions of $6,6-{ }^{2} \mathrm{H}_{2}$-glucose (bolus, $2 \mathrm{mg} / \mathrm{kg}$; continuous, $40 \mu \mathrm{g} / \mathrm{kg}$ per $\mathrm{min}$ ) and ${ }^{2} \mathrm{H}_{5}$-glycerol (bolus, $0.097 \mathrm{mg} / \mathrm{kg}$; continuous, $0.0097 \mathrm{mg} / \mathrm{kg}$ per min). Baseline blood and breath samples were obtained at time $(T)$ $-120 \mathrm{~min}$ for the determination of background isotopic abundances. Thereafter, blood and breath samples were collected every $30 \mathrm{~min}$ for the next $8 \mathrm{~h}$. At times 0,120 and $240 \mathrm{~min}$, the subjects ingested a lemon-flavoured drink containing $0 \cdot 30 \mathrm{~g} / \mathrm{kg}$ per FFM of fructose enriched with ${ }^{13} \mathrm{C}_{6}$ fructose at 1.56625 at $\%{ }^{13} \mathrm{C}$. Energy expenditure and substrate utilisation were continuously monitored by open circuit indirect calorimetry (Deltatrac II; Datex Instruments, Helsinski, Finland). At the end of the study, urine was collected to determine urea nitrogen excretion rate. No additional foods or liquid, except water, were permitted during the test day.

\section{Analytical procedures}

Blood samples were immediately centrifuged at $4^{\circ} \mathrm{C}$ at $3600 \mathrm{rpm}$ for $10 \mathrm{~min}$, and plasma was stored in aliquots at $-20^{\circ} \mathrm{C}$. Plasma glucose was measured with the glucose oxidase method (Glucose Analyser II; Beckman Instruments, Fullerton, CA, USA). Colorimetric methods were used to assess plasma concentrations of NEFA (kit obtained from Wako Chemicals, Freiburg, Germany) and TAG (kit obtained from Biomérieux Vitek, Inc., Lyon, France). Commercial RIA kits were used for the determination of plasma insulin and glucagon (Linco Research, St Charles, MO, USA). $\beta$-Hydroxybutyric acid and lactate concentrations were measured enzymatically using the kits obtained from Boehringer (Mannheim, Germany). Plasma fructose concentrations were measured by $\mathrm{GC}-\mathrm{MS}$; for this purpose, $0.1 \mathrm{ml}$ of $0.55 \mathrm{~mm}$-mannitol was added to $0.2 \mathrm{ml}$ of plasma and vacuum dried. Thereafter, sugars were acetylated with acetic anhydride and pyridine overnight, resuspended in $0.2 \mathrm{ml}$ chloroform and injected into the GC-MS apparatus (Agilent Technologies, Santa Clara, CA, USA) using a HP-5MS 5\% phenyl-methyl siloxane column. Analysis was done in electron impact mode. Ions with $\mathrm{m} / \mathrm{z} 275$ and 361 were monitored for fructose and mannitol, respectively. The ratio of $\mathrm{m} / \mathrm{z}, 361 / 275$ was read against a standard curve prepared using pure fructose ${ }^{(13)}$. Breath ${ }^{13} \mathrm{CO}_{2}$ isotopic enrichment was determined by isotope ratio MS on a Tracermass C/N (SerCon Limited, 
Crewe, Cheshire, UK). Isotopic enrichments of plasma 6,6- ${ }^{2} \mathrm{H}_{2}$-glucose and ${ }^{2} \mathrm{H}_{5}$-glycerol were measured by GC-MS (Agilent Technologies) as described previously ${ }^{(14,15)}$. For the analysis of plasma ${ }^{13} \mathrm{C}$ glucose, plasma glucose was purified by sequential anion/cation exchange chromatography using resins (AG $50 \mathrm{~W}-\mathrm{X} 8$ and $\mathrm{AG} 1-\mathrm{X} 8$; Bio-Rad, Richmond, CA, USA) followed by HPLC ${ }^{(16)}$. Thereafter, ${ }^{13} \mathrm{C}$ abundance in plasma glucose was analysed by continuous flow-isotope ratio MS (Roboprep CN-Tracermass; Europa Scientific, Crewe, UK). Total lipids were extracted from the plasma, and fatty acid methyl esters were prepared from TAG fractions ${ }^{(17)}$. The ratio of ${ }^{13} \mathrm{C}:{ }^{13} \mathrm{C}$ in the fatty acid methyl ester derivatives was ascertained using Delta Plus XP GC-combustion ratio MS (Thermo Electron Corporation, Bremen, Germany). Tricosanoic acid methyl ester was used as an isotopic enrichment standard, and a quality control sample (certified standard of eicosanoic acid and fatty acid methyl esters; Department of Geological Sciences, Indiana University, Bloomington, IN, USA) was run with each set of samples.

\section{Calculations}

Substrate kinetics and ${ }^{13} \mathrm{C}$ glucose appearance. Endogenous glucose production (EGP), i.e. total rate of glucose appearance (Ra), was calculated from plasma $6,6-{ }^{2} \mathrm{H}_{2}$-glucose isotopic enrichment using the non-steady-state equation of Steele as modified by De Bodo et al. ${ }^{(18)}$, using a volume of distribution for glucose of 0.2 times body weight, and a pool fraction of 0.6. Total Ra glycerol was calculated from plasma ${ }^{2} \mathrm{H}_{5}$-glycerol isotopic enrichment as described ${ }^{(19)}$. The contribution of gluconeogenesis from fructose to EGP (EGP (F)) was calculated by monitoring the rate of ${ }^{13} \mathrm{C}$ glucose appearance $^{(20)}$ as follows:

$$
\begin{aligned}
\mathrm{EGP}(\mathrm{F})= & \mathrm{EGP} \times\left({ }^{13} \mathrm{CG}_{2}+{ }^{13} \mathrm{CG}_{1}\right) / 2+p V \times\left(\left(G_{1}+G_{2}\right) / 2\right. \\
& \left.\times\left(\mathrm{CG}_{2}+\mathrm{CG}_{1}\right) /\left(T_{2}-T_{1}\right)\right){ }^{13} \mathrm{CF}
\end{aligned}
$$

where ${ }^{13} \mathrm{CG}$ is the isotopic enrichment of plasma glucose (atom percent (AP) excess), $G$ is the glucose concentration, ${ }^{13} \mathrm{CF}$ is the isotopic enrichment of oral fructose (AP excess) and $T$ is the time. Glucose production from sources other than fructose (EGP (NF), i.e. from glycogenolysis and gluconeogenesis from lactate, amino acids and glycerol) was calculated as follows:

$$
\mathrm{EGP}(\mathrm{NF})=\mathrm{EGP}-\mathrm{EGP}(\mathrm{F}) .
$$

Substrate oxidation and energy expenditure. Net carbohydrate oxidation rates and net lipid oxidation rates were calculated from respiratory gas exchanges and urinary urea nitrogen excretion using the equations of Livesey \& Elia $^{(21)}$. Fructose oxidation was calculated as follows:

$$
\begin{aligned}
&{ }^{13} \mathrm{C} \text { fructose oxidation }(\mathrm{mg} / \mathrm{min}) \\
&=\left({ }^{13} \mathrm{CO}_{2}(\mathrm{AP} \%)(t)-{ }^{13} \mathrm{CO}_{2}(\mathrm{AP} \%)\left(t_{0}\right) /{ }^{13} \mathrm{C}\right. \text { Fruct } \\
&\left.-{ }^{13} \mathrm{CO}_{2}(\mathrm{AP} \%)\left(t_{0}\right)\right) \times\left(\left(V_{\mathrm{CO}_{2}}(t) \times 180\right) /(22 \cdot 29 \times 6 \times c)\right),
\end{aligned}
$$

where ${ }^{13} \mathrm{CO}_{2}(t)$ is the value (AP \%) of the expired $\mathrm{CO}_{2}$ at time $t,{ }^{13} \mathrm{CO}_{2}\left(t_{0}\right)$ is the value $(\mathrm{AP} \%)$ of the expired $\mathrm{CO}_{2}$ at baseline, ${ }^{13} \mathrm{C}$ Fruct is the value (AP \%) of the ingested fructose, $V_{\mathrm{CO}_{2}}(1 / \mathrm{min})$ is the average volume that is expired during the $30 \mathrm{~min}$ period, 180 is the molecular weight of fructose, $1 / 22.29$ is the value used for conversion into moles of $\mathrm{CO}_{2}, 1 / 6$ is the value used for conversion into moles of fructose, $c$ is the $\mathrm{CO}_{2}$ recovery correction factor and chosen to be $0 \cdot 8^{(22)}$.

Non-oxidative fructose disposal (NOFD) was calculated as follows:

$$
\begin{aligned}
\operatorname{NOFD}(\mathrm{g} / \mathrm{kg} \text { FFM })= & \text { fructose load }(\mathrm{g} / \mathrm{kg} \text { FFM }) \\
& - \text { net fructose oxidation }(\mathrm{g} / \mathrm{kg} \text { FFM })
\end{aligned}
$$

De novo lipogenesis. The incorporation of ${ }^{13} \mathrm{C}$ fructose into palmitate of newly synthesised plasma TAG was assessed by monitoring the enrichment of ${ }^{13} \mathrm{C}$ palmitate in VLDL TAG. The ${ }^{13} \mathrm{C}$ enrichment results from fatty acid methyl ester derivatives, expressed as $\delta{ }^{13} \mathrm{C} \%$, were converted to tracer-to-tracee ratio (TTR) using the following equation:

$$
\operatorname{TTR}\left({ }^{13} \mathrm{C}:{ }^{12} \mathrm{C}\right)=\left(\left(\delta^{13} \mathrm{C} \% o\right)+1\right) \times 0 \cdot 0112372 .
$$

The TTR of a baseline measurement, done before ${ }^{13} \mathrm{C}$-labelled fructose administration, was subtracted from each sample's TTR to account for natural abundance ${ }^{(23)}$.

\section{Statistical analysis}

All parameters are expressed as means and standard deviations, except for integrative response of DNL where parameters are expressed as means with their standard errors. Post-fructose responses were calculated as incremental area under the curve (iAUC) using the trapezoidal method by subtracting the fasting value (Microsoft ${ }^{\circledR}$ Office Excel 2003; Microsoft Corporation, Redmond, WA, USA). Relative changes in hormone and substrate concentrations after fructose ingestion were calculated as $100 \times(\mathrm{iAUC} /$ time$) /$ fasting value, and relative changes in substrate oxidation and glucose and glycerol kinetics after fructose ingestion were calculated as mean value $\left(T-30-T_{0}\right)-$ fasting value/fasting value $\times 100$. For each sex, comparisons between fasting and post-fructose values were assessed using a two-sided Wilcoxon signed rank test for paired values. The effects of fructose in males $v$. females were assessed by comparing the fasting parameters and integrated post-fructose parameters using a two-sided Wilcoxon rank sum test for unpaired values. Rates of glycerol appearance were markedly skewed, and were therefore log-transformed before statistical analysis. All calculations were performed with Stata 10 (Stata Corporation, College Station, TX, USA). Statistical significance was set at $P<0 \cdot 05$.

\section{Results}

\section{Anthropometric and fasting metabolic parameters}

Male and female participants did not differ in age or BMI. Weight and \% FFM were higher, and \% FM was lower in males $(P<0.05$; Table 1$)$. As shown in Table 2 , baseline glucose, lactate, TAG, $\beta$-hydroxybutyrate and insulin were not significantly different, whereas NEFA tended to be 
Table 2. Fasting plasma substrate and hormone concentrations of subjects at baseline

(Mean values and standard deviations, $n 18)^{\star}$

\begin{tabular}{|c|c|c|c|c|}
\hline & \multicolumn{2}{|c|}{ Males $(n 9)$} & \multicolumn{2}{|c|}{ Females $(n 9)$} \\
\hline & $\begin{array}{c}\text { Baseline, } \\
\text { mean }\end{array}$ & SD & $\begin{array}{l}\text { Baseline, } \\
\text { mean }\end{array}$ & SD \\
\hline Insulin (pmol/l) & $49 \cdot 8$ & 11.4 & $49 \cdot 8$ & 5.5 \\
\hline Glucose $(\mathrm{mmol} / \mathrm{l})$ & 4.9 & 0.4 & $4 \cdot 8$ & 0.6 \\
\hline Glucagon (pmol/l) & $13 \cdot 3$ & 1.9 & $11.8 \dagger$ & 0.1 \\
\hline Lactate $(\mathrm{mmol} / \mathrm{l})$ & 0.8 & 0.1 & 0.8 & 0.2 \\
\hline Total TAG $(\mathrm{mmol} / \mathrm{l})$ & 0.8 & $0 \cdot 1$ & 0.7 & 0.2 \\
\hline NEFA $(\mathrm{mmol} / \mathrm{l})$ & 0.5 & 0.2 & 0.6 & 0.2 \\
\hline$\beta$-Hyroxybutyrate $(\mathrm{mmol} / \mathrm{l})$ & 0.1 & 0.1 & 0.1 & 0.1 \\
\hline Uric acid $(\mu \mathrm{mol} / \mathrm{l})$ & 345.9 & 53.0 & $248.9 \dagger$ & $63 \cdot 3$ \\
\hline Fructose $(\mu \mathrm{mol} / \mathrm{l})$ & $109 \cdot 0$ & $6 \cdot 7$ & $107 \cdot 9$ & 7.5 \\
\hline
\end{tabular}

${ }^{*}$ Fasting parameters were compared in both sexes using a two-sided Wilcoxon rank sum test for unpaired values.

† Mean values were significantly different from the corresponding values in male subjects $(P<0.05)$.

lower $(P=0 \cdot 08)$. Uric acid and glucagon concentrations were significantly higher in men $(P<0 \cdot 05)$.

\section{Plasma hormone and substrate concentrations}

The relative changes for fructose, glucose, glucagon, TAG and NEFA after fructose ingestion were not significantly different in males $v$. females, whereas the relative increases for insulin, lactate and uric acid were higher $(P<0.05)$ in males (Table 3$)$.

\section{De novo lipogenesis}

Due to a technical problem with the preparation of the drink containing fructose enriched with ${ }^{13} \mathrm{C}_{6}$ fructose during the first test, only eight male subjects were included for obtaining the results pertaining to DNL and ${ }^{13} \mathrm{C}$ glucose appearance. Following fructose ingestion, the ${ }^{13} \mathrm{C}$ enrichment of VLDL palmitate, expressed as TTR, rose significantly in males, but remained close to zero in females. The iAUC for $\mathrm{VLDL}{ }^{13} \mathrm{C}$

Table 3. Relative changes in hormone and substrate concentrations after fructose ingestion, expressed as the percentage of baseline levels (Mean values and standard deviations, $n 18)^{\star}$

\begin{tabular}{lrrrrr}
\hline & \multicolumn{2}{c}{ Males (n 9) } & & \multicolumn{2}{c}{ Females (n 9) } \\
\cline { 2 - 3 } & \multicolumn{1}{c}{ Mean } & SD & & Mean & SD \\
\hline Insulin (\%) & 35.83 & 24.34 & & $13.26 \dagger$ & 12.49 \\
Glucose (\%) & 5.94 & 4.22 & & 4.87 & 7.04 \\
Glucagon (\%) & -0.68 & 7.40 & & -0.21 & 7.13 \\
Lactate (\%) & 76.27 & 28.57 & & $36.34 \dagger$ & 28.42 \\
Total TAG (\%) & -5.28 & 6.63 & & -3.45 & 9.89 \\
NEFA (\%) & -32.92 & 17.61 & & -24.42 & 12.47 \\
B-Hyroxybutyrate (\%) & -44.72 & 28.98 & & -47.54 & 17.69 \\
Uric acid (\%) & 3.69 & 2.40 & & $1.07 \dagger$ & 2.00 \\
Fructose (\%) & 93.32 & 45.23 & & 72.10 & 30.63 \\
& & & & &
\end{tabular}

iAUC, incremental area under the curve.

* The effects of fructose in males $v$. females were assessed by comparing the integrated post-fructose parameters using a two-sided Wilcoxon rank sum test for unpaired values. Relative changes in hormone and substrate concentrations after fructose ingestion were calculated as $100 \times(\mathrm{iAUC} / \mathrm{time}) /$ fasting value.

† Mean values were significantly different from the corresponding values in male subjects $(P<0.05)$. palmitate was significantly higher in males than in females $(P<0 \cdot 05$; Fig. 1).

\section{Glucose and glycerol kinetics and ${ }^{13} \mathrm{C}$ glucose appearance}

Isotopic enrichments of plasma ${ }^{13} \mathrm{C}$ glucose and of $6,6-{ }^{2} \mathrm{H}_{2}$ glucose and ${ }^{2} \mathrm{H}_{5}$-glycerol, which were used for the calculation of substrate kinetics, are shown in Figs. 2 and 3.

Fasting glycerol Ra tended to be higher in females when expressed per kg FFM, but it was similar in males and females when normalised for FM (Fig. 3). Fructose ingestion significantly decreased glycerol $\mathrm{Ra}$ in males (baseline glycerol $\mathrm{Ra} / \mathrm{kg}$ FFM $\times$ min: 3.04 v. 1.94 after fructose ingestion, $P<0.05$; Fig. 3), but not in females (3.79 v. 3.19). Suppression of glycerol $\mathrm{Ra}$ expressed as relative decrease over the $6 \mathrm{~h}$ period following fructose ingestion was blunted in females $(-41.3$ (SD 15.0) in males $v$. $-10 \cdot 2$ (SD 22.9) in females; Table 3), resulting in higher post-fructose glycerol Ra (glycerol $\mathrm{Ra} / \mathrm{kg}$ FFM $\times$ min: 3.19 in females $v$. 1.94 in males). When values were normalised for FM, glycerol Ra was still found to be decreased after fructose ingestion in males, but not in females.

Fasting glucose $\mathrm{Ra}$, normalised for FFM, was slightly higher in females than in males (glucose Ra/kg FFM $\times$ min: 2.56 in males $v .3 .01$ in females, $P<0.05$; Fig. 5). Following
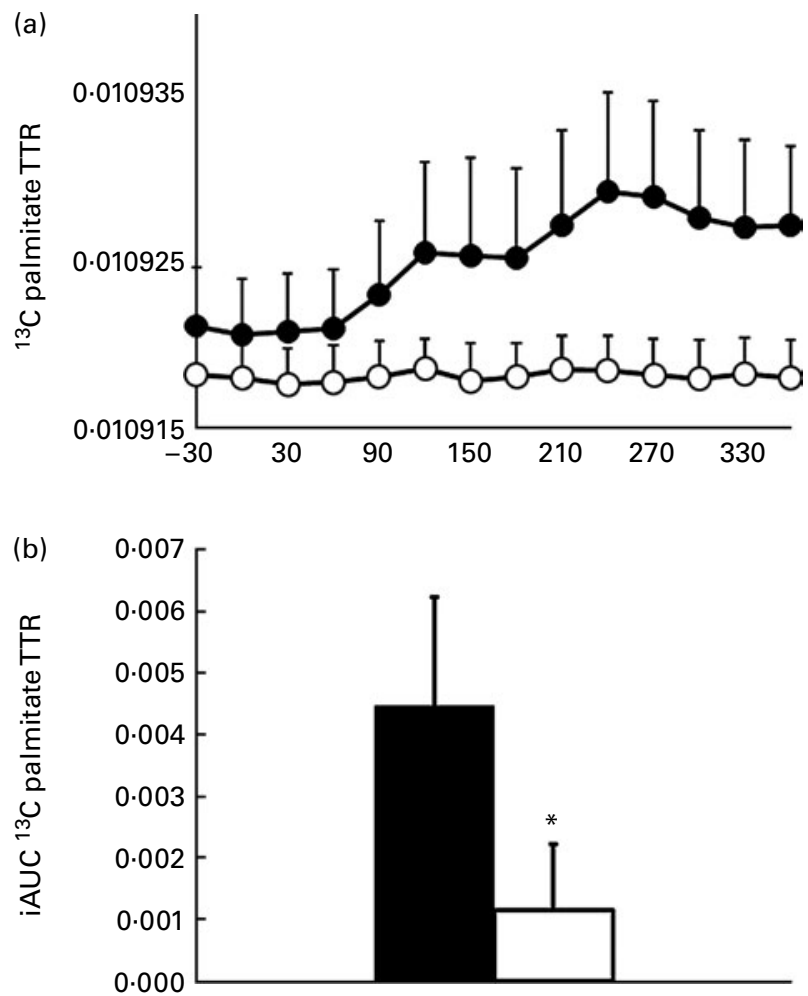

Fig. 1. (a) Mean (SD) time course of the changes in ${ }^{13} \mathrm{C}$ enrichment of VLDL palmitate in males $(\bullet, n 8)$ and females $(O, n 9)$. (b) Incremental area under the curve (iAUC) of ${ }^{13} \mathrm{C}$ palmitate tracer-to-tracee ratio (TTR) after ingestion of fructose in males $(\square, n 8)$ and females $(\square, n 9)$. Statistical significance is only indicated in the figure for differences in integrated responses between males and females. The comparison of iAUC in males $v$. females was assessed using a two-sided Wilcoxon rank sum test for unpaired values. ${ }^{*}$ Mean values were significantly different when compared with males $(P<0.05)$ 

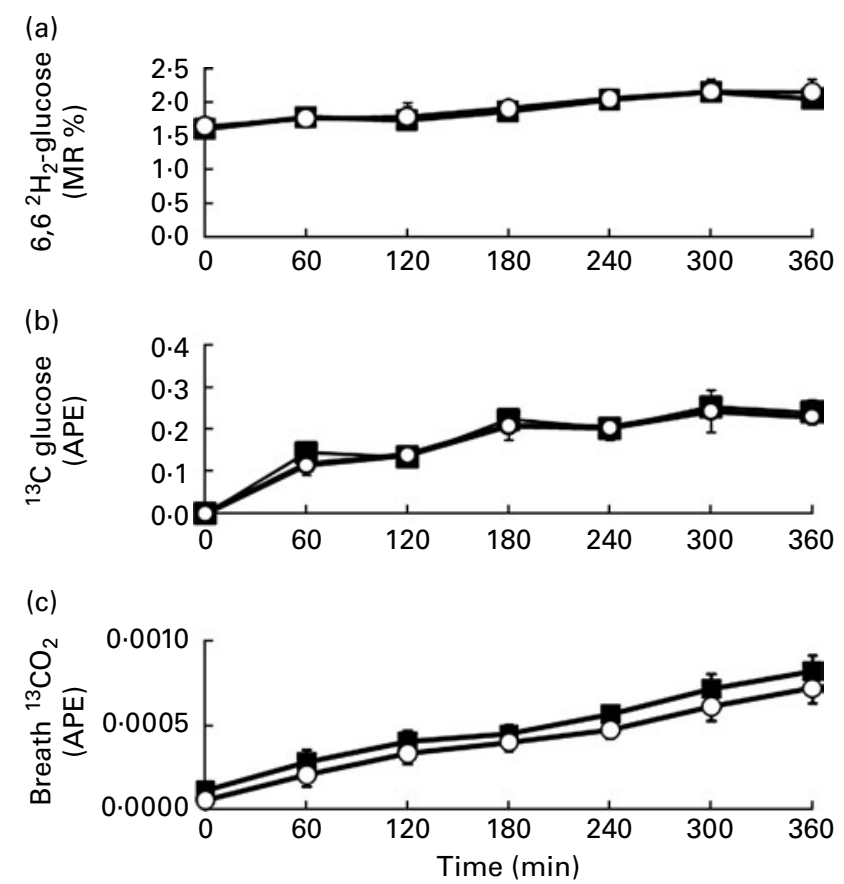

Fig. 2. Mean (SD) time course of the changes in plasma $6,6-{ }^{2} \mathrm{H}_{2}$-glucose (a), ${ }^{13} \mathrm{C}$ glucose (b) and breath ${ }^{13} \mathrm{CO}_{2}$ (c) after ingestion of fructose in males $(\square)$ and females $(O, n 9)$, except for ${ }^{13} \mathrm{C}$ glucose and breath ${ }^{13} \mathrm{CO}_{2}$ where the number of males is 8 . Fructose oral loads $(3 \times 0.30 \mathrm{~g} / \mathrm{kg}$ fat-free mass) were ingested at time 0,120 and $240 \mathrm{~min}$. MR, molar ratio; APE, atom percent excess.

fructose ingestion, glucose Ra decreased slightly over time (Fig. 4). Systemic appearance of ${ }^{13} \mathrm{C}$ glucose, corresponding to gluconeogenesis from ${ }^{13} \mathrm{C}$ fructose, expressed as $\mathrm{EGP}(\mathrm{F})$, represented 44.01 (SD 3.2) and 41.93 (SD 2.3) \% of the total glucose Ra (NS) in males and females, respectively. Glucose appearance from sources other than fructose (i.e. corresponding to glycogenolysis and gluconeogenesis from lactate, amino acids and glycerol), expressed as EGP(NF), was also similar in both sexes (Fig. 4). Fructose conversion into plasma glucose, cumulated over $6 \mathrm{~h}$, represented 37.4 (SD 2.1) \% of the ingested fructose in males and $28.86 \%$ (SD 8.1) in females, $P<0 \cdot 05$.

\section{Net carbohydrate and lipid oxidation and energy expenditure}

In basal conditions, net carbohydrate oxidation was similar in both sexes, but net lipid oxidation was higher in females $(P<0 \cdot 05)$. Net lipid oxidation following fructose ingestion was significantly suppressed (net lipid oxidation in $\mathrm{mg} / \mathrm{kg}$ FFM $\times$ min: 0.67 after fructose ingestion $v .0 .78$ at baseline, $P<0.05)$, whereas net carbohydrate oxidation increased significantly in males (net carbohydrate oxidation in $\mathrm{mg} / \mathrm{kg}$ FFM $\times$ min: $2 \cdot 26$ after fructose ingestion $v .1 .80$ at baseline, $P<0.05)$; in females, in contrast, fructose ingestion did not significantly alter carbohydrate and lipid oxidation (Fig. 5). Fructose oxidation rate calculated from breath ${ }^{13} \mathrm{CO}_{2}$ was similar in both sexes, and accounted for 42.9 (SD 3.7) and 43.0 (SD 4.5$) \%$ of the ingested fructose in males and females, respectively.

The relative change in energy expenditure after fructose ingestion, expressed as the percentage of baseline levels, was higher in males than in females and represented $3 \cdot 10$ (SD 2.21) and 0.06 (SD 2.63)\% $(P<0.05$; Table 4). The iAUC for RQ was significantly larger in males than in females $(P<0 \cdot 05$; Fig. 6).

\section{Discussion}

Fructose oxidation, gluconeogenesis and glycogen storage (mainly in the liver) are known to be the major pathways of disposal of an oral fructose load in human subjects ${ }^{(24-26)}$. Of these, total fructose oxidation (including splanchnic fructose oxidation and indirect peripheral oxidation of glucose) was assessed by monitoring the rate of ${ }^{13} \mathrm{CO}_{2}$ production, and it represented about $43-44 \%$ of the ingested fructose load, without a difference between males and females. Conversion of fructose into plasma glucose was also evaluated by monitoring the systemic appearance of plasma ${ }^{13} \mathrm{C}$-labelled glucose, and it was similar in males and females. Finally,
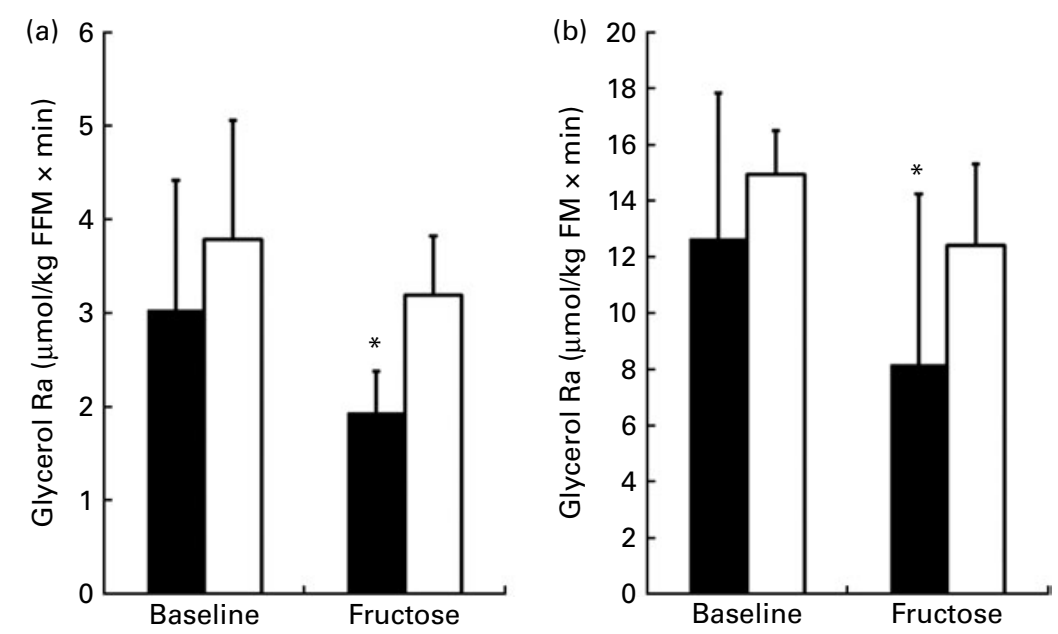

Fig. 3. Mean (SD) glycerol rate of appearance (glycerol Ra) normalised for fat-free mass (FFM; a) and fat mass (FM; b) at baseline and after fructose ingestion. For each sex, comparisons between fasting and post-fructose values were assessed using a two-sided Wilcoxon signed rank test for paired values. ${ }^{\star}$ Mean values were significantly different when compared with baseline $(P<0.05)$. $\mathbf{\square}$, Males; $\square$, females. 


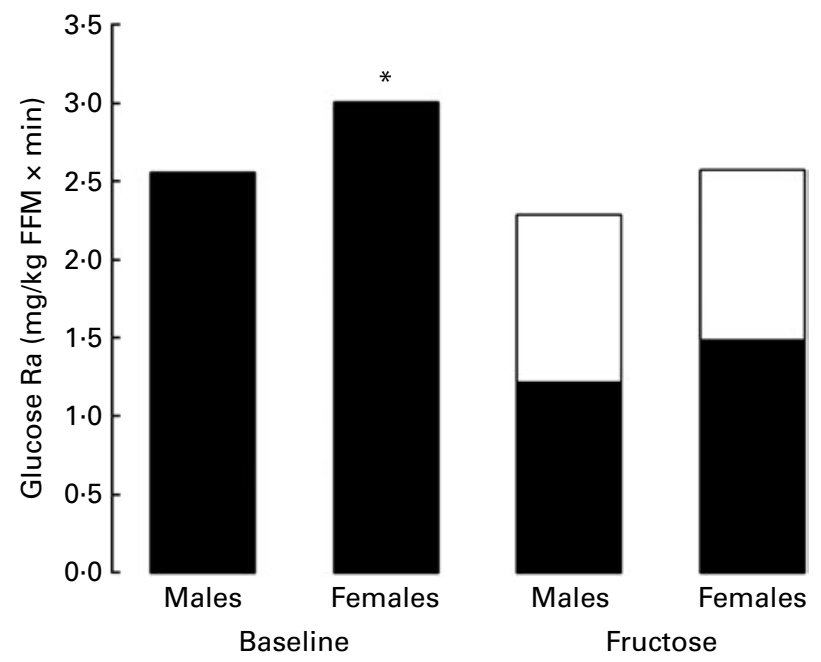

Fig. 4. Mean glucose rate of appearance (glucose Ra) normalised for fat-free mass (FFM) at baseline and after fructose ingestion. Black bars represent endogenous glucose production (glucose $\mathrm{Ra}$ and other than fructose to endogenous glucose production ( $\mathbf{\square}, \mathrm{EGP}(\mathrm{NF})$ ), and white bars represent rates of exogenous (oral) fructose appearance (fructose to EGP $(\square$, EGP $(F)$ ). The effects of fructose in males $v$. females were assessed by comparing fasting parameters and post-fructose parameters using two-sided Wilcoxon rank sum test for unpaired values. * Mean values were significantly different when compared with males $(P<0.05)$.

glycogen storage was not directly assessed. However, NOFD, calculated as the difference between the amount of fructose ingested and net carbohydrate oxidation, is a close approximation of glycogen synthesis, because net carbohydrate oxidation would include any fructose conversion into fat ${ }^{(27)}$. This was not different in males and females. Thus, the quantitatively major pathways of fructose disposal were similar in both sexes.

In previous studies, we and others have reported that short-term fructose consumption is associated with a rapid increase in fasting and post-prandial plasma TAG concentrations $^{(23,28-30)}$. Fructose is the most potent lipogenic substrate in the $\operatorname{diet}^{(28,31,32)}$. Although fat synthesis from fructose is quantitatively minor compared with other pathways of fructose disposal, it can nonetheless have a profound impact on plasma and tissue lipids. Fructose ingestion increases hepatic malonyl-CoA ${ }^{(33)}$, which can increase intra-hepatic fatty acid availability both directly, by stimulating de novo fatty acid synthesis, and indirectly, by inhibiting carnitine palmitoyltransferase-1, and hence intra-hepatic fatty acid oxidation. In a previous study, we have indeed observed that fractional hepatic DNL was positively correlated with plasma VLDL TAG concentrations after the ingestion of a short-term high-fructose diet. Furthermore, inhibition of DNL by a supplementation with long-chain PUFA of marine origin led to a proportionate suppression of VLDL TAG ${ }^{(28)}$. Based on these observations, it appears reasonable to speculate that fructose-induced DNL is instrumental in eliciting at least some of the adverse metabolic effects of fructose. This raised the hypothesis that fructose may stimulate hepatic DNL to a lesser extent in females than in males. The present results support this hypothesis. We indeed observed a significant incorporation of ${ }^{13} \mathrm{C}$-labelled palmitate in VLDL TAG, together with a significant suppression of whole-body lipid oxidation in males, whereas these two effects were conspicuously absent in females. This strongly suggests that females had less fructose-induced DNL. Moreover, we observed a much larger increase in the RQ of males after fructose ingestion, consistent with the suppression of lipid oxidation and an increased conversion of carbohydrate into fat ${ }^{(27,34)}$. Differences in fatty acid synthesis between males and females did not, however, mirror changes in fructose oxidation, gluconeogenesis or glycogen storage, most likely because only a small amount of fructose is converted into fatty acids.

In addition to stimulating more DNL in males than in females, fructose ingestion also differentially affected wholebody lipid metabolism in males $v$. females. Fructose ingestion is known to acutely suppress plasma NEFA and whole-body lipid oxidation. This is best explained by the significant, albeit modest stimulation of insulin secretion elicited by
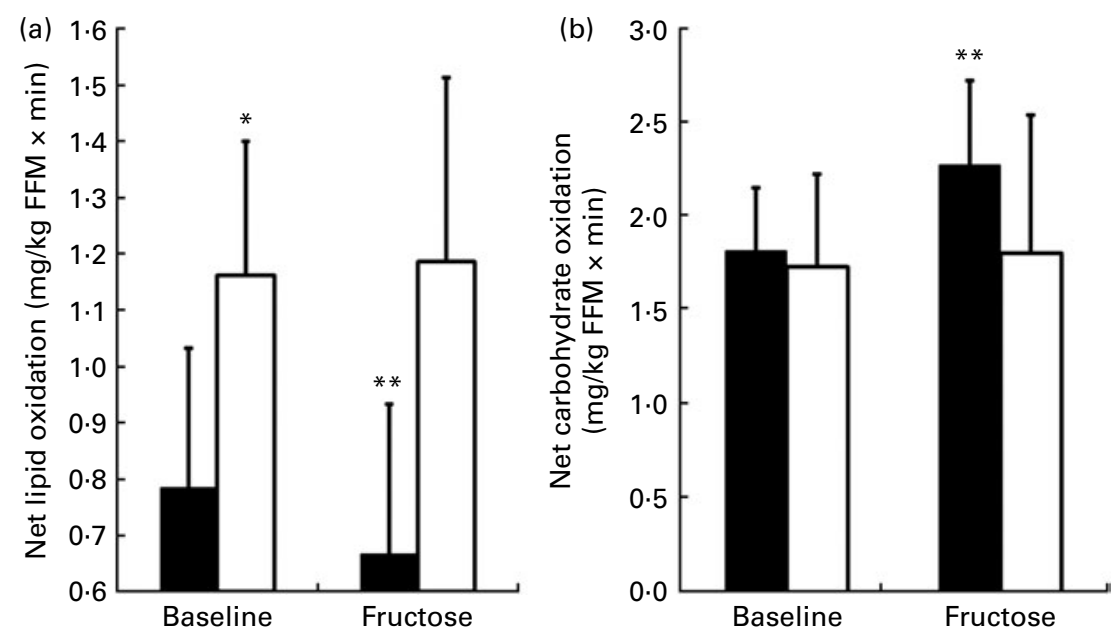

Fig. 5. Net carbohydrate oxidation ( $\mathrm{mg} / \mathrm{kg}$ FFM $\times \mathrm{min}$ ) and net lipid oxidation ( $\mathrm{mg} / \mathrm{kg}$ FFM $\times$ min) at baseline and after fructose ingestion. Values are means and standard deviations. For each sex, comparisons between fasting and post-fructose values were assessed using a two-sided Wilcoxon signed rank test for paired values. ${ }^{*}$ Mean values were significantly different when compared with baseline $(P<0.05)$. The effects of fructose in males $(\square) v$. females $(\square)$ were assessed by comparing the fasting parameters using a two-sided Wilcoxon rank sum test for unpaired values. * Mean values were significantly different when compared with males $(P<0.05)$. 
Table 4. Relative changes in glucose and glycerol kinetics after fructose ingestion, expressed as the percentage of baseline levels

(Mean values and standard deviations, $n 18)^{*}$

\begin{tabular}{|c|c|c|c|c|}
\hline & \multicolumn{2}{|c|}{ Males $(n 9)$} & \multicolumn{2}{|c|}{ Females $(n 9)$} \\
\hline & Mean & SD & Mean & SD \\
\hline $\begin{array}{l}\text { Net carbohydrate } \\
\text { oxidation (\%) }\end{array}$ & 28.65 & 19.05 & $2 \cdot 41$ & $28 \cdot 40$ \\
\hline Net lipid oxidation (\%) & $-16 \cdot 43$ & $15 \cdot 72$ & $1.34 \dagger$ & 14.07 \\
\hline Energy expenditure (\%) & 2.68 & $2 \cdot 84$ & $2 \cdot 30$ & 3.30 \\
\hline $\mathrm{RQ}(\%)$ & $3 \cdot 10$ & $2 \cdot 21$ & $0.06 \dagger$ & 2.63 \\
\hline Ra glucose (\%) & -4.98 & 7.58 & $-13 \cdot 28 \dagger$ & 6.37 \\
\hline Log Ra glycerol (\%) & -41.31 & 15.02 & $-10 \cdot 20 t$ & 22.85 \\
\hline
\end{tabular}

Ra, rate of glucose appearance.

* The effects of fructose in males $v$. females were assessed by comparing the fasting parameters using a two-sided Wilcoxon rank sum test for unpaired values. Relative changes in hormone and substrate concentrations after fructose ingestion were calculated as mean values after fructose ingestion - baseline value) $/ 100 \times$ baseline value.

$\dagger$ Mean values were significantly different from the corresponding values in male subjects $(P<0.05)$.

fructose, which efficiently inhibits adipose tissue lipolysis ${ }^{(35)}$. Glycerol rate of appearance, which, in the absence of exogenous fat metabolism, mainly reflects adipose tissue lipolysis, was more suppressed in males following fructose ingestion. Although part of the difference may be due to the physiologically higher body fat content of females, the glycerol appearance rate normalised for FM remained less suppressed after fructose ingestion in females. This may be explained by the somewhat higher (but NS) insulin response elicited by fructose in males.

The present results point to differences in lipid metabolism in adipose tissues and in the liver of females compared with males. The mechanisms involved in this difference have not been specifically assessed in the present study, and can only be speculated. Several observations strongly support the role of oestrogen in this process. Thus, a lesser increase in plasma TAG after fructose ingestion is observed in pre-menopausal women, but not in post-menopausal women ${ }^{(5)}$. In rodents, sex differences in metabolic responses to fructose can be eliminated by oophorectomy and restored

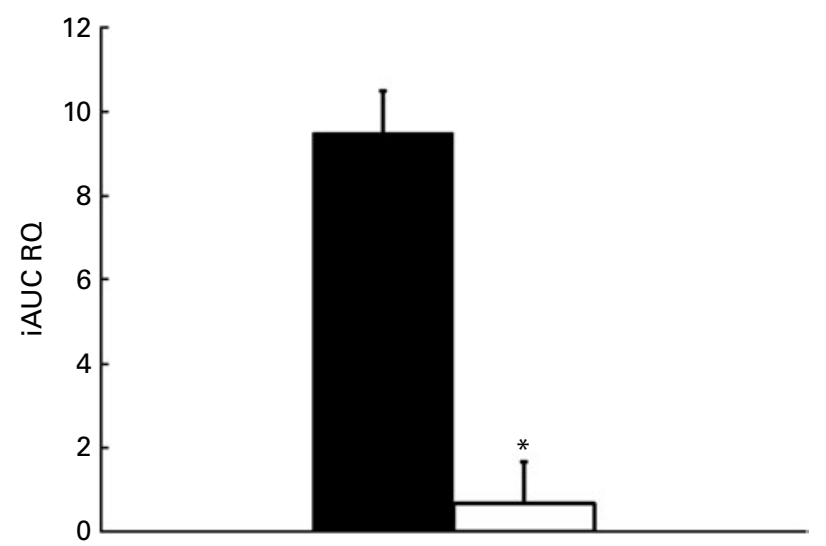

Fig. 6. Incremental area under the curve (iAUC) for $R Q$ after fructose ingestion. The comparison of iAUC in males $(\boldsymbol{\square}) v$. females $(\square)$ was assessed using a two-sided Wilcoxon rank sum test for unpaired values. * Mean values were significantly different when compared with males $(P<0.05)$. by oestrogen substitution ${ }^{(6,7)}$. Our observation points to a dual effect of oestrogens, which stimulate adipose tissue lipolysis while decreasing hepatic DNL. The effect of oestrogen on adipose tissues has indeed been documented by several studies: adipocytes from female mice are known to have increased lipogenic and lipolytic rates compared with those from male mice ${ }^{(36-39)}$. Human in vivo experiments have also demonstrated that oestrogens increase fatty acid availability and oxidation ${ }^{(40,41)}$. In post-menopausal patients, oestrogen replacement therapy reduced the expression of key genes involved in fatty acid synthesis and fat storage, including acetyl-CoA carboxylase, fatty acid synthase and PPAR $\gamma^{(42)}$. Regarding the hepatic effects of oestrogens, to our knowledge, no data are available in human subjects. Oestrogen treatment of female rats, however, reduced hepatic acetyl-CoA carboxylase activity ${ }^{(43)}$. Furthermore, oophorectomy decreased the expression of PPAR $\alpha$ and increased that of sterol regulatory element binding protein-1c, and increased liver TAG content, consistent with a switch towards fat synthesis upon oestrogen withdrawal ${ }^{(44)}$. Oestrogen treatment also reversed hepatic steatosis in male rats made deficient in $\operatorname{aromatase}^{(45)}$.

The present study has several limitations which have to be kept in mind. First, hepatic DNL was qualitatively assessed by monitoring the incorporation of ${ }^{13} \mathrm{C}$ administered as ${ }^{13} \mathrm{C}_{6}$ fructose into VLDL palmitate over time. This is a relatively crude method, which does not allow a quantitative assessment of fatty acid synthesis due to the absence of steady-state conditions and of a simultaneous measurement of VLDL kinetics. Secondly, this method provides an estimate of fractional hepatic DNL, i.e. the fraction of VLDL palmitate issued from de novo synthesis; it is therefore possible that this value was somewhat underestimated in females than in males due to a higher NEFA flux in females. Finally, the fructose load administered was normalised for FFM because the latter is the best predictor of whole-body metabolism. However, this led to a significantly higher administration of fructose per kilogram predicted liver weight in males than in females. This relative hepatic fructose overloading may explain the higher lactate and uric acid responses in males; it may also contribute to a more important stimulation of hepatic DNL in males.

\section{Conclusion}

The present data indicate that oral doses of fructose differentially affect hepatic DNL, adipose tissue lipolysis and lipid oxidation in males and females. This may be due to the effects of female sex hormone on hepatic and adipose metabolism. However, it remains to be assessed whether this sex difference in fructose metabolism confers a significant protection against the potentially harmful effects of fructose in women.

\section{Acknowledgements}

We thank the staff of the Département de Physiologie de Lausanne and of the Cardiomet Clinical Investigation Center for their excellent assistance, and all the volunteers for their participation. The present study was supported by grant no. 310030_121995 from the Swiss National Foundation for Science to L. T.; B. A. F. and K. N. F. were supported by 
the Integrated Project 'Hepatic and adipose tissue functions in the metabolic syndrome' and by the European Commission LSHM-CT-2005-018734 contract no. 018734. The authors' contributions are as follows: C. T., D. J.-D., G. C., K.-A. L. and L. T. designed the study and performed the clamp experiments. P. S. and B. A. F. performed incorporation of ${ }^{13} \mathrm{C}$ into palmitate and plasma glucose and breath $\mathrm{CO}_{2}$ measurement. C. T., D. J.-D., V. L. and L. T. analysed the data. C. T. wrote the draft manuscript, and D. J.-D., V. L., B. A. F., G. C., K.-A. L., M. B., K. N. F., P. S. and L. T. reviewed and edited the manuscript. None of the authors has any conflict of interest.

\section{References}

1. Tappy L \& Le KA (2010) Metabolic effects of fructose and the worldwide increase in obesity. Physiol Rev 90, 23-46.

2. Berneis KK \& Krauss RM (2002) Metabolic origins and clinical significance of LDL heterogeneity. J Lipid Res $\mathbf{4 3}$, 1363-1379.

3. Aeberli I, Zimmermann MB, Molinari L, et al. (2007) Fructose intake is a predictor of LDL particle size in overweight schoolchildren. Am J Clin Nutr 86, 1174-1178.

4. Melanson KJ, Zukley L, Lowndes J, et al. (2007) Effects of high-fructose corn syrup and sucrose consumption on circulating glucose, insulin, leptin, and ghrelin and on appetite in normal-weight women. Nutrition 23, 103-112.

5. Stanhope KL, Griffen SC, Bair BR, et al. (2008) Twentyfour-hour endocrine and metabolic profiles following consumption of high-fructose corn syrup-, sucrose-, fructose-, and glucose-sweetened beverages with meals. Am J Clin Nutr 87, 1194-1203.

6. Bantle JP, Raatz SK, Thomas W, et al. (2000) Effects of dietary fructose on plasma lipids in healthy subjects. Am J Clin Nutr 72, 1128-1134.

7. Couchepin C, Le KA, Bortolotti M, et al. (2008) Markedly blunted metabolic effects of fructose in healthy young female subjects compared with male subjects. Diabetes Care 31, 1254-1256.

8. Macdonald I (1966) Influence of fructose and glucose on serum lipid levels in men and pre- and postmenopausal women. Am J Clin Nutr 18, 369-372.

9. Vasudevan H, Xiang H \& McNeill JH (2005) Differential regulation of insulin resistance and hypertension by sex hormones in fructose-fed male rats. Am J Physiol Heart Circ Physiol 289, H1335-H1342.

10. Galipeau D, Verma S \& McNeill JH (2002) Female rats are protected against fructose-induced changes in metabolism and blood pressure. Am J Physiol Heart Circ Physiol 283, $\mathrm{H} 2478-\mathrm{H} 2484$.

11. Song D, Arikawa E, Galipeau D, et al. (2004) Androgens are necessary for the development of fructose-induced hypertension. Hypertension 43, 667-672.

12. Durnin JV \& Womersley J (1974) Body fat assessed from total body density and its estimation from skinfold thickness: measurements on 481 men and women aged from 16 to 72 years. Br J Nutr 32, 77-97.

13. Petersen KF, Laurent D, Yu C, et al. (2001) Stimulating effects of low-dose fructose on insulin-stimulated hepatic glycogen synthesis in humans. Diabetes 50, 1263-1268.

14. Schricker T, Albuszies G, Kugler B, et al. (1994) Determination of glycerol turnover by stable-isotope technique in humans: a new $\left[1,1,2,3,3-{ }^{2} \mathrm{H}_{5}\right]$ glycerol derivative for mass-spectrometry analysis. Nutrition 10, 342-345.
15. Wolfe R (editor) (1992) Radioactive and Stables Isotope Tracers in Biomedicine: Principles and Practice of Kinetics Analysis. New York: Wiley-Liss.

16. Gay LJ, Schneiter P, Schutz Y, et al. (1994) A non-invasive assessment of hepatic glycogen kinetics and post-absorptive gluconeogenesis in man. Diabetologia 37, 517-523.

17. Bickerton AS, Roberts R, Fielding BA, et al. (2007) Preferential uptake of dietary fatty acids in adipose tissue and muscle in the postprandial period. Diabetes 56, 168-176.

18. DeBodo RC, Steele R, Altszuler N, et al. (1963) On the hormonal regulation of carbohydrate metabolism: studies with ${ }^{14} \mathrm{C}$ glucose. Recent Prog Horm Res 19, 445-448.

19. Romijn JA, Coyle EF, Sidossis LS, et al. (1993) Regulation of endogenous fat and carbohydrate metabolism in relation to exercise intensity and duration. Am J Physiol 265, $3 \mathrm{Pt} 1$, E380-E391.

20. Proietto J, Rohner-Jeanrenaud F, Ionescu E, et al. (1987) Non-steady-state measurement of glucose turnover in rats by using a one-compartment model. Am J Physiol 252, 1 Pt 1, E77-E84.

21. Livesey G \& Elia M (1988) Estimation of energy expenditure, net carbohydrate utilization, and net fat oxidation and synthesis by indirect calorimetry: evaluation of errors with special reference to the detailed composition of fuels. Am J Clin Nutr 47, 608-628.

22. Allsop JR, Wolfe RR \& Burke JF (1978) Tracer priming the bicarbonate pool. J Appl Physiol 45, 137-139.

23. Chong MF, Fielding BA \& Frayn KN (2007) Mechanisms for the acute effect of fructose on postprandial lipemia. Am J Clin Nutr 85, 1511-1520.

24. Delarue J, Normand S, Pachiaudi C, et al. (1993) The contribution of naturally labelled ${ }^{13} \mathrm{C}$ fructose to glucose appearance in humans. Diabetologia 36, 338-345.

25. Tounian P, Schneiter P, Henry S, et al. (1994) Effects of infused fructose on endogenous glucose production, gluconeogenesis, and glycogen metabolism. Am $J$ Physiol 267, 5 Pt 1, E710-E717.

26. Neese RA, Schwarz JM, Faix D, et al. (1995) Gluconeogenesis and intrahepatic triose phosphate flux in response to fasting or substrate loads. Application of the mass isotopomer distribution analysis technique with testing of assumptions and potential problems. J Biol Chem 270, 14452-14466.

27. Frayn $\mathrm{KN}$ (1983) Calculation of substrate oxidation rates in vivo from gaseous exchange. J Appl Physiol 55, 628-634.

28. Faeh D, Minehira K, Schwarz JM, et al. (2005) Effect of fructose overfeeding and fish oil administration on hepatic de novo lipogenesis and insulin sensitivity in healthy men. Diabetes 54, 1907-1913.

29. Le KA, Faeh D \& Stettler R (2006) A 4-wk high-fructose diet alters lipid metabolism without affecting insulin sensitivity or ectopic lipids in healthy humans. Am J Clin Nutr 84, 1374-1379.

30. Abdel-Sayed A, Binnert C, Le KA, et al. (2008) A high-fructose diet impairs basal and stress-mediated lipid metabolism in healthy male subjects. Br J Nutr 100, 393-399.

31. Parks EJ, Skokan LE, Timlin MT, et al. (2008) Dietary sugars stimulate fatty acid synthesis in adults. $J$ Nutr 138, 1039-1046.

32. Stanhope KL \& Havel PJ (2008) Fructose consumption: potential mechanisms for its effects to increase visceral adiposity and induce dyslipidemia and insulin resistance. Curr Opin Lipidol 19, 16-24.

33. Van ben Berghe G (1978) Metabolic effects of fructose in the liver. Curr Top Cell Regul 13, 97-135.

34. Brown CM, Dulloo AG, Yepuri G, et al. (2008) Fructose ingestion acutely elevates blood pressure in healthy young humans. Am J Physiol Regul Integr Comp Physiol 294, R730-R737. 
35. Tappy L, Randin J, Felber J, et al. (1986) Comparison of thermogenic effect of fructose and glucose in normal humans. Am J Physiol 250, 6 Pt 1, E718-E724.

36. Pujol E, Rodriguez-Cuenca S, Frontera M, et al. (2003) Genderand site-related effects on lipolytic capacity of rat white adipose tissue. Cell Mol Life Sci 60, 1982-1989.

37. Mittendorfer B, Horowitz JF \& Klein S (2001) Gender differences in lipid and glucose kinetics during short-term fasting. Am J Physiol Endocrinol Metab 281, E1333-E1339.

38. Macotela Y, Boucher J, Tran TT, et al. (2009) Sex and depot differences in adipocyte insulin sensitivity and glucose metabolism. Diabetes 58, 803-812.

39. Cooke PS \& Naaz A (2004) Role of estrogens in adipocyte development and function. Exp Biol Med (Maywood) 229, 1127-1135.

40. Halkes CJ, van Dijk H, Verseyden C, et al. (2003) Gender differences in postprandial ketone bodies in normolipidemic subjects and in untreated patients with familial combined hyperlipidemia. Arterioscler Thromb Vasc Biol 23, 1875-1880.

41. Blaak E (2001) Gender differences in fat metabolism. Curr Opin Clin Nutr Metab Care 4, 499-502.

42. Lundholm L, Zang H, Hirschberg AL, et al. (2008) Key lipogenic gene expression can be decreased by estrogen in human adipose tissue. Fertil Steril 90, 44-48.

43. Diamant YZ, Neuman S \& Shafrir E (1975) Effect of chorionic gonadotropin, triamcinolone, progesterone and estrogen on enzymes of placenta and liver in rats. Biochim Biophys Acta 385, 257-267.

44. Paquette A, Wang D, Jankowski M, et al. (2008) Effects of ovariectomy on PPAR alpha, SREBP-1c, and SCD-1 gene expression in the rat liver. Menopause 15, 1169-1175.

45. Hewitt KN, Pratis K, Jones ME, et al. (2004) Estrogen replacement reverses the hepatic steatosis phenotype in the male aromatase knockout mouse. Endocrinology 145, 1842-1848. 